\title{
PASSED: Calculate Power and Sample Size for Two Sample Tests
}

by Jinpu Li, Ryan P. Knigge, Kaiyi Chen, Emily V. Leary

\begin{abstract}
Power and sample size estimation are critical aspects of study design to demonstrate minimized risk for subjects and justify the allocation of time, money, and other resources. Researchers often work with response variables that take the form of various distributions. Here, we present an $\mathrm{R}$ package, PASSED, that allows flexibility with seven common distributions and multiple options to accommodate sample size or power analysis. The relevant statistical theory, calculations, and examples for each distribution using PASSED are discussed in this paper.
\end{abstract}

\section{Introduction}

Power and sample size estimation are critical aspects of study design to demonstrate minimized risk for subjects and justify the allocation of time, money, and other resources (Jones et al., 2003). A number of $\mathrm{R}$ packages for power analysis have been developed over the years. The samplesize (Scherer, 2016) package provides the calculation of sample size for the Student's t-test and the Wilcoxon-Mann Whitney test for categorical data. The TrialSize (Zhang et al., 2013) package implements the power analysis described in Chow et al. (2007), including power and sample size calculations for different study designs. Most recently, the simglm (LeBeau, 2019) package presents a simulation approach for power analysis that allows for the specification of missing data, unbalanced designs, and different random error distributions of generalized linear models.

Moreover, researchers often work with response variables that can take the form of a variety of distributions. For example, the proportion of thromboembolism after surgery in different treatment groups can be modeled using the binomial distribution or length of inpatient stay after an orthopedic procedure can be modeled using the Poisson distribution (Plessl et al., 2020). Some of the R packages or functions are designed to calculate the power and sample size for the variables following a certain distribution. The base package stats (R Core Team, 2016) provides such functions for normal (Gaussian) and binomially distributed variables, and the situations of unequal sample sizes are extended by packages pwr (Champely et al., 2017), MESS(Ekstrøm, 2012), pwr2ppl(Aberson, 2019), and WebPower (Zhang and Mai, 2018). The package MKmisc (Kohl, 2021) further adds a function for the comparison of negative binomial distributions. However, none of these packages provide a comprehensive power analysis toolkit capable of calculating power or sample sizes for the test of two-sample means or ratios when the responses have other common distributions (Table 1).

\begin{tabular}{|c|c|c|c|c|c|c|c|}
\hline Package & Binomial & Normal & $\begin{array}{l}\text { Negative Bi- } \\
\text { nomial }\end{array}$ & Geometric & Poisson & Beta & Gamma \\
\hline PASSED & $x$ & $x$ & $x$ & $x$ & $x$ & $x$ & $x$ \\
\hline stats & $x^{*}$ & $x^{*}$ & & & & & \\
\hline pwr & $\mathrm{x}$ & $x^{* *}$ & & & & & \\
\hline WebPower & $\mathrm{x}$ & $x^{* *}$ & & & & & \\
\hline MESS & $\mathrm{x}$ & $\mathrm{x}$ & & & & & \\
\hline pwr2ppl & $\mathrm{x}$ & $\mathrm{x}$ & & & & & \\
\hline MKmisc & & & $\mathrm{x}$ & $\mathrm{x}$ & & & \\
\hline
\end{tabular}

Table 1: The comparison among PASSED and other available packages.

Here, we present an R package, PASSED, that performs power and sample size analyses for the following distributions: binomial, negative binomial, geometric, Poisson, normal (Gaussian), beta, and gamma distributions. Distributions, which had existing functions or R infrastructure for sample size and power calculations were included to streamline these calculations. However, calculations for the beta, Poisson, and gamma distributions were developed specifically for inclusion in PASSED. In the following sections, we will discuss the motivating examples, relevant statistical theory, and 
calculations for each distribution using PASSED.

\section{PASSED: R Package Description}

All functions in this package can be used to compute the power for a specific study design (e.g., given sample sizes) or to estimate specific parameter values (e.g., sample sizes) necessary to obtain a target power. The specific function of interest will depend on the type of outcome variable and the data distribution. All functions output an object of class power. htest that details the specified parameters of the test and the estimated parameter set as NULL.

\section{Binomial}

The binomial distribution is useful when modeling the number of successes in a sequence of independent and identically distributed Bernoulli trials. One example which uses data modeled using a binomial distribution is the proportion of blood transfusion that has occurred during surgery. The need for blood transfusion during surgery is an important consideration during surgical planning and particularly for surgical trials. LEITE et al. (2020) applied a logistic regression with binomial outcomes to model the rate of blood transfusions after the introduction of Tranexamic acid in knee arthroplasty.

Hypothesis Testing two-sample proportions is commonly considered in research designs when the outcome follows a binomial distribution. Let $x_{i j}$ be a binary response from the $j$ th subject in the $i t h$ group, $j=1, \ldots, n_{i}, i=1,2$. It is assumed that $x_{i j}$ are independent Bernoulli random variables with proportion $p_{i}$,

$$
x_{i j} \sim \operatorname{Bernoulli}\left(p_{i}\right)
$$

Two hypothesis frameworks are considered for power and sample size calculations, which correspond to either a one-sided or two-sided test:

$$
H_{0}: p_{1}=p_{2} \text { vs. } H_{a}: p_{1} \neq p_{2}(\text { two }- \text { sided })
$$

or

$$
H_{0}: p_{1}=p_{2} \text { vs. } H_{a}: p_{1}>(<) p_{2}(\text { one }- \text { sided })
$$

Algorithm A binomial asymptotic test statistic was first proposed by Pearson (1900). Fleiss et al. (1980) provided an explicit formula to calculate the corresponding sample sizes for the test:

$$
n_{1}=\frac{\left[z_{\frac{\alpha}{2}} \sqrt{(r+1) \bar{p} \bar{q}}+z_{\beta} \sqrt{r p_{1} q_{1}+p_{2} q_{2}}\right]^{2}}{r d^{2}}(t w o-\text { sided })
$$

or

$$
n_{1}=\frac{\left[z_{\alpha} \sqrt{(r+1) \bar{p} \bar{q}}+z_{\beta} \sqrt{r p_{1} q_{1}+p_{2} q_{2}}\right]^{2}}{r d^{2}}(\text { one }- \text { sided }),
$$

where $r=n_{2} / n_{1}, d=p_{2}-p_{1}, q_{1}=1-p_{1}, q_{2}=1-p_{2}, \bar{p}=\frac{n_{1} p_{1}+n_{2} p_{2}}{n_{1}+n_{2}}, \bar{q}=1-\bar{p}$, and $z_{x}$ denotes the probability that a standard normal deviate is greater than $x$. To obtain the power, this equation can be re-written as:

$$
\begin{aligned}
& z_{\beta}=\frac{\sqrt{r n_{1}}|d|-z_{\frac{\alpha}{2}} \sqrt{(r+1) \bar{p} \bar{q}}}{\sqrt{r p_{1} q_{1}+p_{2} q_{2}}}(\text { two }- \text { sided }) \\
& z_{\beta}=\frac{\sqrt{r n_{1}}|d|-z_{\alpha} \sqrt{(r+1) \bar{p} \bar{q}}}{\sqrt{r p_{1} q_{1}+p_{2} q_{2}}}(\text { one }- \text { sided })
\end{aligned}
$$

And, thus, the power can be derived as:

$$
\begin{aligned}
& \text { Power }=\operatorname{Pr}\left(Z<\frac{z_{\frac{\alpha}{2}} \sqrt{(r+1) \bar{p} \bar{q}}-\sqrt{r n_{1}}|d|}{\sqrt{r p_{1} q_{1}+p_{2} q_{2}}}\right)(\text { two }- \text { sided }) \\
& \text { Power } \left.=\operatorname{Pr}\left(Z<\frac{z_{\alpha} \sqrt{(r+1) \bar{p} \bar{q}}-\sqrt{r n_{1}}|d|}{\sqrt{r p_{1} q_{1}+p_{2} q_{2}}}\right) \text { (one }- \text { sided }\right)
\end{aligned}
$$

As a result, the target power, required sample sizes $\left(n_{1}\right.$ and $\left.n_{2}\right)$, significance level $(\alpha)$, or the proportions ( $p_{1}$ and $p_{2}$ ) can be obtained once all other remaining parameters are known (Fleiss et al., 1980). To optimize the sample size allocation, please refer to the discussion in Brittain and Schlesselman (1982). 
Function The power_Binomial() function is useful when testing for differences among two sample proportions when the data follow a binomial distribution. This function uses the algorithm described above. The arguments for power_Binomial() are as follows:

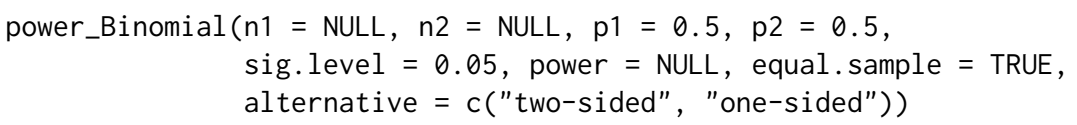

Sample sizes for each group are designated as n1 and n2. If sample sizes for both groups are equal, the argument equal. sample should be set to TRUE, and only a value for $n 1$ is needed. If sample sizes are unequal, equal. sample should be set to FALSE, and values for both $n 1$ and $n 2$ must be specified. When estimating other parameters, the target power must be set with power. The significance level is set with sig. level and has a default value of 0.05 . The probability of success for each group is indicated as $\mathrm{p} 1$ and p2, respectively, with 0.5 as the default value for both. Only one of the parameters of $n 1, n 2, p 1, p 2$, power, or sig. level can be set as NULL. The parameter set as NULL will be estimated based on the other parameter values. The argument al ternative specifies the alternative hypothesis as either "two.sided" (default) or "one. sided".

The power_Binomial() function returns the same results as stats: :power.prop.test() in the equal sample scenario. It also allows power calculations with unequal sample sizes, and the results are identical to MESS: : power_prop_test().

\section{Negative Binomial}

The negative binomial distribution can be used to model the number of successes in a sequence of independent and identically distributed Bernoulli trials before a specified number of failures occurs. Gates et al. (2020) analyzed the probability of positive intraoperative cultures in a population of patients with a history of prior ipsilateral shoulder surgery. The probability of the total number of positive tissue cultures was modeled using a generalized negative binomial mixed model with maximum likelihood estimation and robust standard errors. Using this negative binomial framework, the appropriate sample size and power for such a study can be obtained using the method outlined below.

Hypothesis Consider a sequence of adverse events. Let $x_{i j}$ be the number of events during time $t_{i}$ from the $j$ th subject in the ith group, $j=1, \ldots, n_{i}, i=1,2$. Assuming that $x_{i j}$ are negative binomial random variables with a mean $\mu_{i j}$ and parameter $\theta(\theta>0)$, the probability function of $x_{i j}$ is

$$
P\left(x_{i j}\right)=\frac{\Gamma\left(\theta+x_{i j}\right)}{\Gamma(\theta) x_{i j} !}\left(\frac{\mu_{i j}}{\theta+\mu_{i j}}\right)^{x_{i j}}\left(\frac{\theta}{\theta+\mu_{i j}}\right)^{\theta},
$$

where $n$ ! denotes the product of the integers from 1 to $n$ and $\Gamma(\cdot)$ is the gamma function (Zhu and Lakkis, 2014).

To model the negative binomial outcomes, Hilbe (2011) introduced the negative binomial regression. Zhu and Lakkis (2014) then presented a hypothesis test comparing two negative binomial distributed samples using negative binomial regression, and this is the method used here. In negative binomial regression, $\mu_{i j}$ can be modeled as

$$
\log \left(\mu_{i j}\right)=\log \left(t_{i}\right)+\beta_{0}+\beta_{1} G_{i j}
$$

where $G_{i j}$, the group indicator for subject $\mathrm{j}$, is equal to 0 if $i=1$ for group 1 and is equal to 1 if $i=2$ for group 2. Let $r_{1}$ and $r_{2}$ be the mean rates of events per time unit for groups 1 and 2, which can be expressed as $r_{1}=e^{\beta_{0}}$ and $r_{2}=e^{\beta_{0}+\beta_{1}}$. Then $r_{2} / r_{1}=e^{\beta_{1}}$ can be easily obtained (Zhu and Lakkis, 2014).

To compute the power of the test or determine parameters to obtain target power, two hypothesis frameworks are considered which correspond to either a one-sided or two-sided test:

$$
H_{0}: \frac{r_{2}}{r_{1}}=1 \text { vs. } H_{a}: \frac{r_{2}}{r_{1}} \neq 1(\text { two }- \text { sided })
$$

or

$$
H_{0}: \frac{r_{2}}{r_{1}}=1 \text { vs. } H_{a}: \frac{r_{2}}{r_{1}}>(<) 1(\text { one }- \text { sided })
$$

Algorithm The power and sample size calculation algorithms were developed by Zhu and Lakkis (2014) based on the asymptotic normality of the maximum likelihood estimation of $\beta_{1}$. The power can 
be calculated as:

$$
\text { power }=\Phi\left(\frac{\sqrt{n_{1}}\left|\log \left(\frac{r_{2}}{r_{1}}\right)\right|-z_{\frac{\alpha}{2}} \sqrt{V_{0}}}{\sqrt{V_{1}}}\right)(\text { two }- \text { sided })
$$

or

$$
\text { power }=\Phi\left(\frac{\sqrt{n_{1}}\left|\log \left(\frac{r_{2}}{r_{1}}\right)\right|-z_{\alpha} \sqrt{V_{0}}}{\sqrt{V_{1}}}\right)(\text { one }- \text { sided }),
$$

where $V_{0}$ and $V_{1}$ are the estimates of variance for $\hat{\beta}_{1}$ by $n_{1}$ under $H_{0}$ and $H_{a}$,

$$
\begin{aligned}
& V_{0}=\frac{1}{t_{i}}\left(\frac{1}{\tilde{r}_{1}}+\frac{n_{1}}{n_{2} \tilde{r}_{2}}\right)+\frac{\left(n_{1}+n_{2}\right)}{\theta n_{2}} \\
& V_{1}=\frac{1}{t_{i}}\left(\frac{1}{r_{1}}+\frac{n_{1}}{n_{2} r_{2}}\right)+\frac{\left(n_{1}+n_{2}\right)}{\theta n_{2}},
\end{aligned}
$$

and $\tilde{r}_{i}, i=1,2$ denotes the estimation of the event rate under $H_{0}$ in each group. Zhu and Lakkis (2014) provided three approaches to estimating $\tilde{r}_{i}$ under $H_{0}$ :

Approach 1: using event rate of group 2 (reference group rate)

$$
V_{0}=\frac{1}{t_{i}}\left(\frac{1}{r_{2}}+\frac{n_{1}}{n_{2} r_{2}}\right)+\frac{\left(n_{1}+n_{2}\right)}{\theta n_{2}}
$$

Approach 2: using true rates

$$
V_{0}=\frac{1}{t_{i}}\left(\frac{1}{r_{1}}+\frac{n_{1}}{n_{2} r_{2}}\right)+\frac{\left(n_{1}+n_{2}\right)}{\theta n_{2}}
$$

Approach 3: using maximum likelihood estimation

$$
V_{0}=\frac{1}{t_{i}}\left(\frac{1}{\frac{n_{1} r_{1}+n_{2} r_{2}}{n_{1}+n_{2}}}+\frac{n_{1}}{n_{2} \frac{n_{1} r_{1}+n_{2} r_{2}}{n_{1}+n_{2}}}\right)+\frac{\left(n_{1}+n_{2}\right)}{\theta n_{2}} .
$$

Function The function power_NegativeBinomial() is useful when developing a study design to compare differences in rates when the data follow a negative binomial distribution. Calculations for this function are based on Zhu and Lakkis (2014). The following arguments are used:

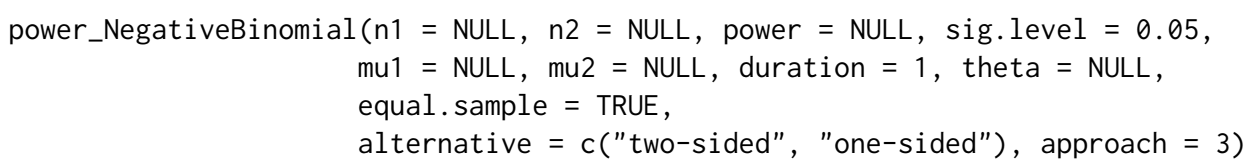

The sample size for each group is specified as $n 1$ and $n 2$, both with default values of NULL. When sample sizes are equal, equal. sample can be set to TRUE, and only $n 1$ must be specified. Otherwise equal. sample is set to FALSE and values must be input for both $\mathrm{n} 1$ and $\mathrm{n} 2$. The power argument is set to NULL unless the target power is specified here and another parameter is set as NULL to be estimated. The significance level for the test is set by sig. level with a default value of 0.05 . The expected rates of events per unit time for each group are denoted as mu1 and mu2, respectively, with the average treatment duration set by duration (default value of 1 ). Theta indicates the $\theta$ parameter of the negative binomial distribution, as noted above. The argument al ternative specifies the alternative hypothesis as either "two.sided" (default) or "one.sided". Lastly, the argument approach can be set as either "1", "2", or "3" (default). These values indicate the selection of one of three procedures for estimating the variance under the null hypothesis for the sample size formula and correspond with Approach 1 (reference group rate), Approach 2 (true rates), and Approach 3 (maximum likelihood estimation) described above. The obtained results match other functions in R such as MKmisc: : power. nb. test().

\section{Geometric}

The geometric distribution can be used to examine the probability of success given a limited number of trials and is considered a special case of the negative binomial distribution. For example, in baseball, the probability of a batter earning a hit before striking out can be compared to that of another batter, using a geometric distribution. 
Hypothesis Let $x_{i j}$ be the number of events during time $t_{i}$ from the $j$ th subject in the $i t h$ group. Assuming that $x_{i j}$ are geometric random variables with a mean $\mu_{i j}$, the probability function of $x_{i j}$ is

$$
P\left(x_{i j}\right)=\left(\frac{\mu_{i j}}{1+\mu_{i j}}\right)^{x_{i j}}\left(\frac{1}{1+\mu_{i j}}\right) .
$$

Referring to Equation 1, this is a special case of the negative binomial where $\theta=1$. Similarly, $\mu_{i j}$ can be modeled as shown in the Section Negative Binomial,

$$
\log \left(\mu_{i j}\right)=\log \left(t_{i}\right)+\beta_{0}+\beta_{1} G_{i j} .
$$

The hypotheses and calculations follow as previously shown in the Section Negative Binomial.

Algorithm The power and sample size calculation formula are the same as the Section Negative Binomial, with $\theta=1$.

Function The function power_Geometric() applies the same algorithm as the function power_NegativeBinomial(), with the same arguments, where the parameter theta is set as 1 . See power_NegativeBinomial() for more details.

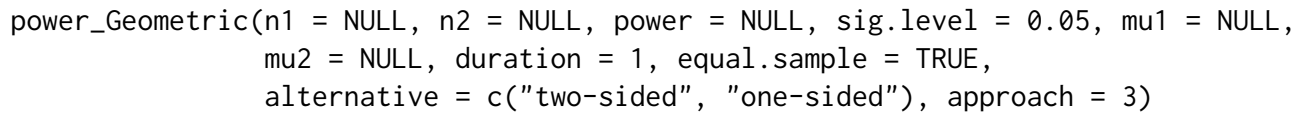

\section{Poisson}

The Poisson distribution can be used to model the number of events occurring in a fixed interval of time or space. In healthcare, length of stay (LOS) is one of many important considerations for interventions, particularly when inpatient hospital stay may vary among treatments. LOS, or other count measurements important to the research study, can be modeled using a Poisson distribution. Plessl et al. (2020) used a Poisson framework to compare LOS for those who were treated with rapid recovery protocols versus standard recovery protocols after total knee arthroplasty. This example can be expanded to the general case as follows.

Hypothesis Let $x_{i j}$ be the number of events during the necessary study time $t_{i}$ from the $j$ th subject in the $i t h$ treatment group, $j=1, \ldots, n_{i}, i=1,2$. This situation is commonly referred to as the equal sampling frame approach (Hutchinson and Holtman, 2005). It is assumed that $x_{i j}$ are Poisson random variables with rate $\lambda_{i}$ such that the probability function of $x_{i j}$ is

$$
P\left(x_{i j}\right)=\frac{t_{i} \lambda_{i} e^{t_{i} \lambda_{i}}}{x_{i j} !},
$$

where $i=1,2$. Then, the total number of events in each group, denoted as $X_{1}$ and $X_{2}$, also follow a Poisson distribution:

$$
X_{i} \sim \operatorname{Poisson}\left(\lambda_{i} t_{i} n_{i}\right)
$$

Four methods have previously been proposed to test the equality of two Poisson rates (Shiue and Bain, 1982; D. Huffman, 1984; Thode, 1997; Gu et al., 2008). The method utilized in the PASSED package was proposed by Gu et al. (2008), which considers the ratio of two Poisson rates, $R$, a pre-specified positive number. The asymptotic test is as follows:

$$
H_{0}: \lambda_{2} / \lambda_{1}=R \text { vs. } H_{a}: \lambda_{2} / \lambda_{1}=R^{\prime} \neq R(\text { two }- \text { sided })
$$

or

$$
H_{0}: \lambda_{2} / \lambda_{1}=R \text { vs. } H_{a}: \lambda_{2} / \lambda_{1}=R^{\prime}>R(\text { one }- \text { sided })
$$

Algorithm The following formula is used in the PASSED package and the details of the derivation are provided in the Appendix.

$$
n_{1}=\frac{\left(\frac{z_{1-\frac{\alpha}{2}} C+z_{\text {power }} D}{A}\right)^{2}-\frac{3}{8}}{\lambda_{1} t_{1}}(\text { two }- \text { sided })
$$


or

$$
n_{1}=\frac{\left(\frac{z_{1-\alpha} C+z_{\text {power }} D}{A}\right)^{2}-\frac{3}{8}}{\lambda_{1} t_{1}}(\text { one }- \text { sided })
$$

where $A=2\left(1-\sqrt{\frac{R}{R^{\prime}}}\right)$,

$B=\lambda_{1} t_{1} n_{1}+3 / 8$,

$C=\sqrt{\frac{R+d}{R^{\prime}}}$

$D=\sqrt{\frac{R^{\prime}+d}{R^{\prime}}}$

$d=t_{1} / t_{2}$.

Function The power_Poisson() function is designed to compute the power or estimate parameters to obtain a target power when testing for a ratio of two Poisson rates. This function applies the asymptotic tests based on normal approximations developed by Gu et al. (2008). The arguments for power_Poisson() are as follows:

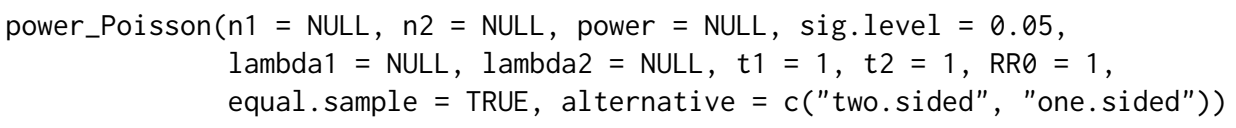

Sample sizes for each group are set with $\mathrm{n} 1$ and $\mathrm{n} 2$. If sample sizes for both groups are equal, the argument equal. sample should be set to TRUE, and only a value for $n 1$ needs to be specified. If sample sizes are unequal, equal. sample should be set to FALSE, and values for both $n 1$ and $n 2$ must be specified. The target power of the test is set with power, and the significance level is set with sig. level (default value of 0.05). The expected rates of events per unit time for each group are denoted as lambda1 and lambda2, respectively, with the average treatment duration set by $\mathrm{t} 1$ and $\mathrm{t} 2$ (default value of 1). Only one of the parameters of $n 1, n 2$, lambda1, lambda2, or power can be set as NULL for the function to run. The parameter set as NULL will be estimated based on the other parameter values. $t 1$ and $t 2$ refer to the specified interval of time (or space) where the events occur. The rate ratio from the null hypothesis is specified as RR 0 . It should be set to 1 when testing for equal Poisson rates. The argument alternative specifies the alternative hypothesis as either "two.sided" (default) or "one.sided".

For the example in Gu et al. (2008), which aims at testing if the risk of coronary heart disease is greater for those with postmenopausal hormone use $(\mathrm{RR} 0=1)$, the event rates for those with and without hormone use are assumed to be 0.2000 and 0.0005 (lambda2 $=0.0020$, lambda $1=0.0005$ ), respectively, during a 2-year time period $(\mathrm{t} 1=\mathrm{t} 2=2)$. Given the sample size for each group as 4295 and $8590(\mathrm{n} 2=4295, \mathrm{n} 1=8590)^{1}$, the power under a significance level of 0.05 can be calculated as follows:

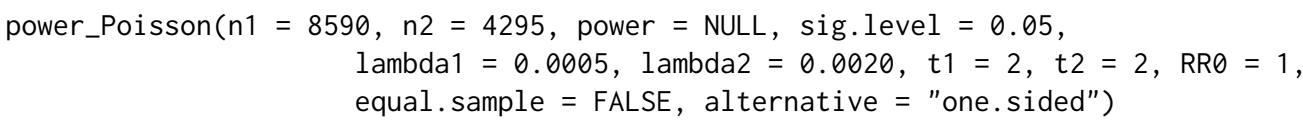

The estimated power is 0.9000147, which matches the results in Gu et al. (2008).

\section{Normal}

The normal distribution is widely used in the natural and social sciences. Age is a common demographic variable recorded during patient care and typically follows a normal distribution. Many surgeons consider demographic variables to evaluate the possible risks of a surgical procedure and assess optimal treatment options for patients. Luan et al. (2020) aimed to identify patients who were suitable for kinematic or mechanical alignment of the knee. To compare these groups, Luan et al. (2020) used the student $\mathrm{t}$-test to compare normally distributed age.

Hypothesis T-tests are widely used to compare two sample means when the data has a normal distribution (Cressie and Whitford, 1986). Let $x_{i j}$ be a continuous response from the $j$ th subject in the $i$ th group, $j=1, \ldots, n_{i}, i=1,2$. It is assumed that $x_{i j}$ are independent, normal random variables with mean $\mu_{i}$ and variance $\sigma_{i}^{2}$ :

$$
x_{i j} \sim \operatorname{Normal}\left(\mu_{i}, \sigma_{i}^{2}\right),
$$

\footnotetext{
${ }^{1}$ the sample sizes are corrected in NCSS Software Manuals 2020 Page 437-14, "Tests for the Ratio of Two Poisson Rates"
} 
then the probability density function of $x_{i j}$ is:

$$
f\left(x_{i j}\right)=\frac{1}{\sqrt{2 \pi \sigma_{i}^{2}}} e^{-\frac{1}{2}\left(\frac{x_{i j}-\mu_{i}}{\sigma_{i}}\right)^{2}},
$$

where $i=1,2$. It can be shown that the mean of each group, denoted as $\bar{x}_{1}$ and $\bar{x}_{2}$, also follows a Normal distribution:

$$
\bar{x}_{i} \sim \operatorname{Normal}\left(\mu_{i}, \frac{\sigma_{i}^{2}}{n_{i}}\right)
$$

To compute the power for a hypothesis test or determine parameters to obtain a target power for hypothesis, the following two scenarios are considered:

$$
H_{0}: \mu_{1}=\mu_{2} \text { vs. } H_{a}: \mu_{1} \neq \mu_{2}(\text { two }- \text { sided })
$$

or

$$
H_{0}: \mu_{1}=\mu_{2} \text { vs. } H_{a}: \mu_{1}>(<) \mu_{2}(\text { one }- \text { sided })
$$

Algorithm Based on the work of Ekstrøm (2012), in the PASSED package, the user can define the sample sizes $\left(n_{1}\right.$ and $\left.n_{2}\right)$ and standard deviations $\left(\sigma_{1}\right.$ and $\left.\sigma_{2}\right)$ of each group directly, rather than set the size ratio $\left(n_{2} / n_{1}\right)$ and standard deviation ratio $\left(\sigma_{2} / \sigma_{1}\right)$. To optimize sample size allocation, please refer to the discussion in Jan and Shieh (2011).

Function The power_Normal() function is useful for developing a study design to test for differences between mean values of two groups when the data follow a normal distribution. This function performs the same operations as pwr.t. test in the pwr package (Champely et al., 2017) but allows for additional parameter modifications. In particular, this function allows for specifying unequal sample sizes and standard deviations across groups. The arguments for power_Normal() are as follows:

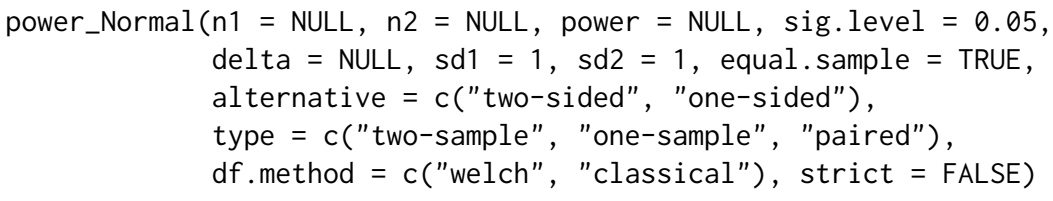

Sample sizes for each group are set with $\mathrm{n} 1$ and $\mathrm{n} 2$. If sample sizes for both groups are equal, the argument equal. sample should be set to TRUE, and only a value for $n 1$ needs to be specified. If sample sizes are unequal, equal. sample must be set to FALSE, and values for both $n 1$ and $n 2$ must be specified. The target power of the test is set with power, and the significance level is set with sig. level (default value of 0.05). delta indicates the difference in means between the two groups, and sd1 and sd2 denote the standard deviations for each group. A default value of 1 is indicated for both sd1 and sd2. The default values for $\mathrm{n} 1, \mathrm{n} 2$, power, and delta are NULL, whereas sd1, sd2, and sig. level have non-NULL default values. Only one of the parameters can be set as NULL. The parameter set as NULL will be estimated based on the other parameter values. The type of $t$-test is indicated by type and set as "two. sample" (default), "one. sample", or "paired". alternative specifies the alternative hypothesis as either "two.sided" (default) or "one.sided". Lastly, df.method indicates the method for calculating the degrees of freedom as either "welch" (default) or "classical". Note that setting strict as TRUE would be applied only in the two-sided case, when the probability of rejection in the opposite direction of the true effect is included, i.e., the alternative hypothesis of the two-sided t-test is $\mu_{1} \neq \mu_{2}$ rather than $\mu_{1}>(<) \mu_{2}$.

The power_Normal() function produces the same results as stats: : power.t.test() for the equal sample size scenario. It also allows power calculations with unequal sample sizes and unequal variances. The results match other functions in R such as MESS: : power_prop_test() and pwr: : pwr.t2n.test().

\section{Beta}

The beta family of continuous probability distributions is ideal for modeling data with right or left skewness and allows the probability density to assume a variety of shapes through two shape parameters (Gupta and Nadarajah, 2004). Disease status is often measured with bounded outcome scores, which take values on a finite range. The distribution of such data is often skewed, rendering the standard analysis methods assuming a normal distribution inappropriate (Hu et al., 2020), and thus, a beta distribution can be utilized. This scenario can be generalized as follows. 
Hypothesis Suppose a sequence of random responses, $x_{i j}$ from the $j$ th subject in the $i t h$ group, takes the form of a continuous proportion that follows a beta distribution, $x_{i j} \sim \operatorname{Bet} a\left(a_{i}, b_{i}\right)$, where $j=1, \ldots, n_{i}, i=1,2$. The probability density function of $x_{i j}$ is:

$$
f\left(x_{i j}\right)=\frac{\Gamma\left(a_{i}+b_{i}\right)}{\Gamma\left(a_{i}\right) \Gamma\left(b_{i}\right)} x_{i j}^{a_{i}-1}\left(1-x_{i j}\right)^{b_{i}-1},
$$

where $0 \leq x_{i j} \leq 1, a_{i}>0, b_{i}>0$, and $i=1,2$. When analyzing continuous proportions as a response variable, the standard shape parameters of a beta density, $a_{i}$ and $b_{i}$, are often not directly observable. Ferrari and Cribari-Neto (2004) developed a class of beta regression models which utilize an alternative parameterization of the beta density function based on the mean, $\mu_{i}$, and an unknown precision parameter, $\phi_{i}$. Suppose $\mu_{i}=a_{i} /\left(a_{i}+b_{i}\right)$ and $\phi_{i}=a_{i}+b_{i}$, then the beta density function can be expressed in terms of $\mu_{i}$ and $\phi_{i}$ as below:

$$
f\left(x_{i j}\right)=\frac{\Gamma\left(\phi_{i}\right)}{\Gamma\left(\mu_{i} \phi_{i}\right) \Gamma\left(\left(1-\mu_{i}\right) \phi_{i}\right)} x_{i j}^{\mu_{i} \phi_{i}-1}\left(1-x_{i j}\right)^{\left(1-\mu_{i}\right) \phi_{i}-1} ;
$$

For beta regression, $\mu_{i}$ can be modeled as

$$
g\left(\mu_{i}\right)=\beta_{0}+\beta_{1} G_{i j},
$$

where $G_{i j}$, the group indicator for subject $j$, is equal to 0 if $i=1$ for group 1 and is equal to 1 if $i=2$ for group 2, and $g(\cdot)$ denotes the link function. The PASSED package includes the capability for the following link functions and their respective forms:

- Logit: $g(\mu)=\log \left[\frac{\mu}{(1-\mu)}\right]$

- Probit: $g(\mu)=\Phi^{-1}(\mu)$

- Complementary log-log: $g(\mu)=\log [-\log (1-\mu)]$

- Log: $g(\mu)=\log (\mu)$

- Log-log: $g(\mu)=-\log [-\log (\mu)]$

The equality of means $\mu_{i}$ is equivalent to $\beta_{1}=0$. The objective is to compute the power of the test or determine minimum sample sizes to obtain a target power for the needed hypothesis. A two-sided hypothesis framework is considered for power and sample size calculations:

$$
H_{0}: \mu_{1}-\mu_{2}=0 \text { vs. } H_{a}: \mu_{1}-\mu_{2} \neq 0
$$

Algorithm The mean and variance of $x_{i j}$, denoted as $\mu_{i}$ and $\sigma_{i}^{2}$, can be obtained using:

$$
\mu_{i}=\frac{a_{i}}{a_{i}+b_{i}}
$$

and

$$
\sigma_{i}^{2}=\frac{a_{i} b_{i}}{\left(a_{i}+b_{i}\right)^{2}\left(a_{i}+b_{i}+1\right)} .
$$

Incorporating the definition of the precision parameter $\phi_{i}$, the following equations can be derived:

$$
\begin{gathered}
a_{i}=\mu_{i} \phi_{i}=\mu_{i}\left(\frac{\mu_{i}\left(1-\mu_{i}\right)}{\sigma_{i}^{2}}-1\right) ; \\
b_{i}=\left(1-\mu_{i}\right) \phi_{i}=\left(1-\mu_{i}\right)\left(\frac{\mu_{i}\left(1-\mu_{i}\right)}{\sigma_{i}^{2}}-1\right) .
\end{gathered}
$$

To calculate power, a simulation approach is used. Parameters $\mu_{i}$ and $\phi_{i}$ are first estimated using the given mean and variance, then they are used to obtain the original beta parameters, $a_{i}$ and $b_{i}$, following Equations 2 and 3 . The response variable is simulated for each distribution, Beta $\left(a_{1}, b_{1}\right)$ and $\operatorname{Bet} a\left(a_{2}, b_{2}\right)$, with the given sample size. If any simulated response is equal to zero or one, the following transformation is applied to each response value from both distributions: $(x(n-1)+0.5) / n$, where $x$ is the response value and $n$ is the sample size (Smithson and Verkuilen, 2006).

Values for the simulated response from both distributions are merged together, along with the group indicator ( 0 for group 1 and 1 for group 2$)$. Subsequently, a beta regression model is built using the specified link type (Cribari-Neto and Zeileis, 2010). A Wald test is performed on the simulated model, testing the null hypothesis that $\beta_{1}$ is equal to 0 . The $p$-values are recorded for each test and the 
simulation is repeated $M$ times. The power is calculated as:

$$
\text { power }=\frac{\text { Number of } \mathrm{p} \text {-values less than } 0.05}{M} .
$$

Let ss denote sample size, then the generic power/sample size relationship can be formally expressed as:

$$
\text { power }=\mathrm{f}(\mathrm{ss})
$$

Assuming the response variable follows a beta distribution, $f(\cdot)$ is continuous on the interval $(0,1)$ and increases monotonically. Consequently, the power_Beta function uses the bisection method to obtain the minimum sample size, $s s_{0}$, through a sequence of steps for each iteration (Chernick and Liu, 2002). For each target power, power 0 , upper and lower sample size bounds, $s s_{u}$ and $s s_{l}$, which satisfy $f\left(s s_{l}\right)<$ power $_{0}<f\left(s s_{u}\right)$ are established using a two-sample t-test performed with the base function power.t.test (R Core Team, 2016). Although power.t. test assumes normality, it is useful to generate starting values for $s s_{u}$ and $s s_{l}$.

The sequence of steps for each iteration is as follows:

1. Compute the midpoint $s s_{\text {mid }}=$ floor $\left(\frac{s s_{l}+s s_{u}}{2}\right)$ of interval $\left[s s_{l}, s s_{u}\right]$. floor $(\cdot)$ denotes retaining the integer part of a number.

2. Calculate power at the midpoint, $s s_{\text {mid }}$, using the simulation described for the power calculations above.

3. If $f\left(s s_{\text {mid }}\right) \geq$ power $_{0}$ and $s s_{\text {mid }}-s s_{l} \leq 1$, then return $s s_{\text {mid }}$ and stop iterating.

4. Examine the sign of $f\left(s s_{\text {mid }}\right)-$ power $_{0}$. If negative, then replace $s s_{l}$ with $s s_{\text {mid }}$, otherwise replace $s s_{u}$ with $s s_{\text {mid }}$ so that $f\left(s s_{l}\right)<$ power $_{0} \leq f\left(s s_{u}\right)$.

Repeat the process until iteration stops. The output minimum sample size, $s s_{0}$, is the minimum integer such that $f\left(s s_{0}\right) \geq$ power $_{0}$.

Function The power_Beta() function is framed to test differences between mean values for two groups, assuming the response variable follows a beta distribution in each group. It can be used to compute the power or to estimate the required sample sizes to obtain a target power. In particular, this function allows for specifying unequal sample sizes and standard deviations across groups. The arguments for power_Beta() are as follows:

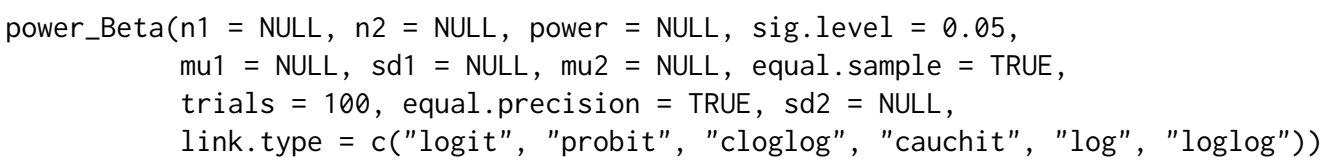

Sample sizes for each group are set with $\mathrm{n} 1$ and $\mathrm{n} 2$. If sample sizes for both groups are equal, the argument equal. sample should be set to TRUE, and only a value for $n 1$ or power needs to be specified. If sample sizes are unequal, equal. sample should be set to FALSE, and values for both $n 1$ and $n 2$ must be specified. The target power of the test is set with power, and the significance level is set with sig. level (default value of 0.05 ). Only one of the parameters of $n 1, n 2$, or power can be NULL. The mean and standard deviation for the null distribution are denoted by mu1 and sd1. Analogously, the mean and standard deviation for the alternative distribution can be specified by mu2 and sd2. Note that equal. precision=FALSE should be used to set the standard deviation for the alternative distribution, meaning the precision parameters are assumed to be unequal. Otherwise, option sd2 would be ignored. The option trials indicates the number of trials in the simulation. A default number of trials (i.e., 100) is recommended to get a rough estimate of other parameters (e.g., sd2), since the computational time is dependent upon the number of trials in the simulation. Once an appropriate range of other values is determined, the number of trials should be increased (e.g., trials $=1000)$ to calculate precise power and sample size estimates. The default link function is the logit link but can be changed using link. type with the following options: "logit", "probit", "cloglog", "log", "loglog", to denote the logit, probit, complementary $\log -\log$, log, and log-log link functions, respectively.

\section{Gamma}

The gamma distribution is widely used to fit lifetime data because its flexibility in shape can vary from extremely positively skewed to almost symmetric (Casella and Berger, 2002). Hong et al. (2020) provide an example of modeling data using the gamma distribution to test the association of patient-provider cost discussion with out-of-pocket spending among cancer survivors. The data (i.e., out-of-pocket spending in cancer care) have an obvious skewness which is not normally distributed; and therefore, 
the two-sample t-test is not suitable for this purpose. Alternatively, gamma models can be used to test the difference of average total out-of-pocket spending between the patients with and without a patient-provider cost discussion.

Hypothesis Currently, there is no explicit formula to calculate the power comparing two gamma random variables. Let $x_{i j}$ be a continuous response from the $j$ th subject in the ith group $, j=1, \ldots, n_{i}$, $i=1$, 2 . It is assumed that $x_{i j}$ are gamma random variables with scale $\lambda_{i}$ and shape $\delta_{i}$ so that the probability density function can be written as

$$
f\left(x_{i j}=x\right)=\left(\frac{\lambda_{i}}{\Gamma\left(\delta_{\mathrm{i}}\right)}\right) x^{\delta_{i}-1} e^{-\lambda_{i} x}
$$

The mean of $\operatorname{Gamma}\left(\lambda_{i}, \delta_{i}\right)$ can be obtained using $\mu_{i}=\delta_{i} / \lambda_{i}$. Shiue and Bain (1983) developed a test of two equal gamma means with unknown common shape parameter, such that

$$
H_{0}: \mu_{1}=\mu_{2}=\mu \text {. }
$$

This can be re-written as $H_{0}: \delta=\lambda_{1} \mu=\lambda_{2} \mu$, some $\delta>0$. This can then be tested using an F distribution based on the ratio of the mean of a random sample from two gamma distributions. In 1988, Shiue et al. (1988) extended this to the unknown and unequal shape parameter scenarios. However, this extension can be slightly conservative and problematic for smallscale parameters. More recently, Chang et al. (2011) provided a computational approach using a variant of the parametric bootstrap method, used here, in which the shape parameters are completely unknown and unequal. In this characterization, the hypothesis is two-sided and is of the form $H_{0}: \delta_{i}=\lambda_{i} \mu$, some $\mu>0$ or equivalently, for two means,

$$
H_{0}: \frac{\delta_{1}}{\lambda_{1}}=\frac{\delta_{2}}{\lambda_{2}} \text { vs. } H_{a}: \frac{\delta_{1}}{\delta_{1}} \neq \frac{\delta_{2}}{\lambda_{2}} .
$$

This can be expressed as a scalar value function, $\eta$, such that

$$
H_{0}^{*}: \eta=\sum_{i=1}^{2}\left(\beta_{i}-\bar{\beta}\right)^{2}=0 \text { vs. } H_{a}^{*}: \eta>0,
$$

where $\beta_{i}=\ln \left(\mu_{i}\right)$ and $\bar{\beta}=\sum_{i=1}^{2} \frac{\beta_{i}}{2}$.

Algorithm The power and sample size calculation algorithm adapted for PASSED was developed by Chang et al. (2011). This computational approach performs best when the restricted maximum likelihood estimate of $\eta$ behaves as approximately normal or as a sum of squared normals.

Function The power_Gamma() function is used to compute the power or estimate sample sizes to obtain a target power when testing for differences among two sample means when the data follow a gamma distribution. This function used a parametric bootstrap method addressed by Chang et al. (2011). The arguments for power_Gamma() are as follows:

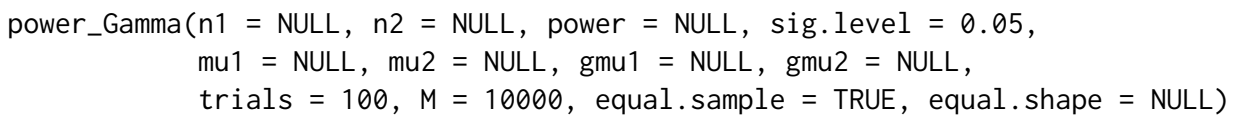

Sample sizes for each group are set with $\mathrm{n} 1$ and $\mathrm{n} 2$. If sample sizes for both groups are equal, the argument equal. sample should be set to TRUE, and only a value for $\mathrm{n} 1$ or power needs to be specified. If sample sizes are unequal, equal. sample should be set to FALSE, and values for both $n 1$ and $n 2$ must be specified. The target power of the test is set with power, and the significance level is set with sig. level (default value of 0.05 ). Only one of the parameters of $n 1, n 2$, or power can be set as NULL. The parameter set as NULL will be estimated based on the other parameter values. The arithmetic means for each group are indicated by mu1 and mu2, while gmu1 and gmu2 denote the geometric mean for each group, respectively. Option trials specifies the number of trials in the simulation, and the number of generated samples in every single trial is identified by M. A small number of trials (e.g., using the default value 100) is recommended to get a rough estimate of power or sample size since the computational time is dependent upon the number of trials in the simulation. To obtain a reasonable result, a greater value (e.g., 10000) should be used for both trials and M. The assumption of equal shape parameters should be tested before the comparison of two sample means if equal. shape is set as NULL (default value is NULL). Otherwise, the test to determine equal shape is skipped (when equal. shape is set to be TRUE or FALSE). 
For example, Schickedanz and Krause (1970) presented the weekly rainfall data for the seasons of fall and winter. The arithmetic/geometric means are $0.3684 / 0.2075$ for winter $(n=57)$ and $0.7635 / 0.3630$ for fall $(n=51)$. Using a significance level of 0.05 , the power can be calculated as follows:

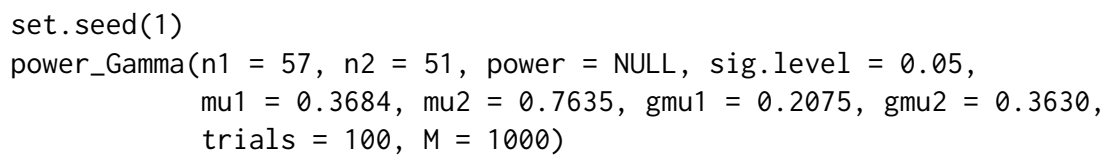

The estimated power is 1.00, which matches the result in Schickedanz and Krause (1970).

\section{Application of PASSED}

In this section, we provide an example power analysis and sample size calculation implemented with PASSED. We propose a hypothetical study to test an intervention protocol designed to reduce the percentage of residents at nursing facilities who develop new or worsening pressure ulcers, known as bedsores.

The Skilled Nursing Facility Quality Reporting Program (SNF-QRP) provider dataset contains information on pressure ulcer rates among nursing home facilities across the US. In this scenario, half of the participating nursing homes will implement the intervention protocol (treatment group), and the other half will constitute a control group, without a change in protocol, to determine if the new intervention reduces rates of pressure ulcers. We consider the following hypotheses for the study:

- $H_{0}$ : There is no difference in pressure ulcer rates among nursing home facilities between control and treatment groups.

- $H_{a}$ : There is a difference in pressure ulcer rates among nursing home facilities between control and treatment groups.

\section{Sample Size Determination}

In this example, we use the mean and standard deviation of the SNF-QRP variable, "percentage of SNF residents with pressure ulcers that are new or worsened" for the control group mu1 and sd1, 0.0174 and 0.0211 , respectively. A $25 \%$ decrease in the proportion of patients that develop new or worsening pressure ulcers is considered significant and results in the target alternative mean, mu2, equal to 0.0131. To determine the appropriate number of facilities necessary in the control and treatment groups, we first use power_Beta to estimate the minimum sample size with target power equal to 0.8 . The power_Beta is chosen because this proportion is defined on the interval $[0,1]$ and right-skewed. The default value of link. type is used, trials is set at 1000 , and equal precision in the control and treatment groups is assumed. This analysis can be fine-tuned through additional iterations of power_Beta by modifying the number of trials. The output is given below:

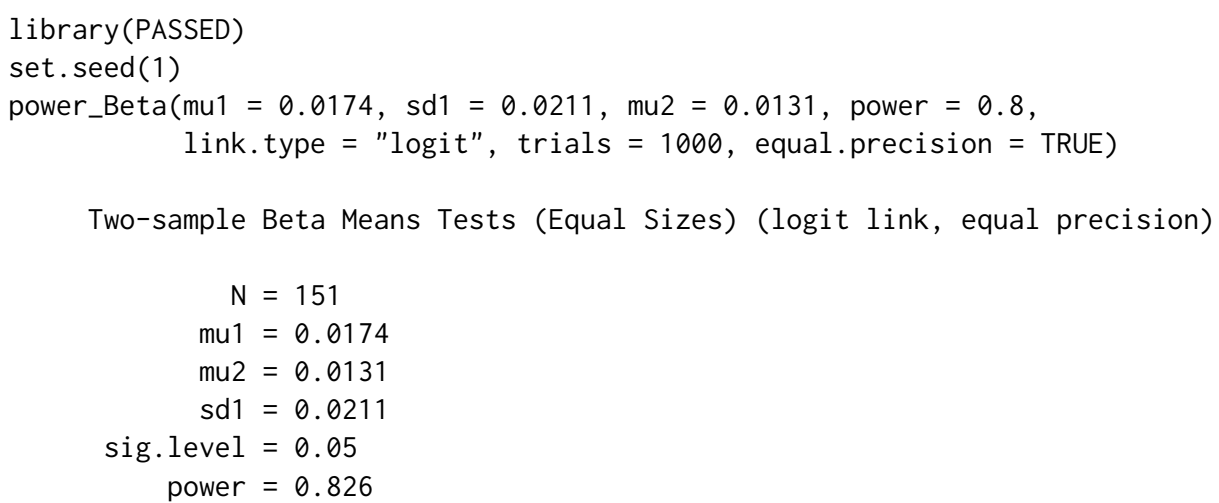

NOTE: $\mathrm{N}$ is number in *each* group

The obtained result indicates that 302 nursing home facilities (151 facilities for each group) are necessary to demonstrate the difference between pressure ulcer rates among the control and treatment groups, with a significance level of 0.05 and power of 0.80 . 


\section{Comparison with T-Test}

To further assess the appropriate number needed in the control and treatment groups, we then use 0.0120 to 0.0140 to evaluate a range of target means that encompass the target's alternative mean of 0.0131 , with expected sample sizes of over 100 nursing homes per group. As a comparison, we also calculate the power using a two-sided t-test under the same scenario, using the function power_Normal. The true difference in means, delta, is set as the difference of mu1 and mu2, and the alternative standard deviation is assumed to be equal to sd1. The output for this example is displayed below, assuming equal precision.

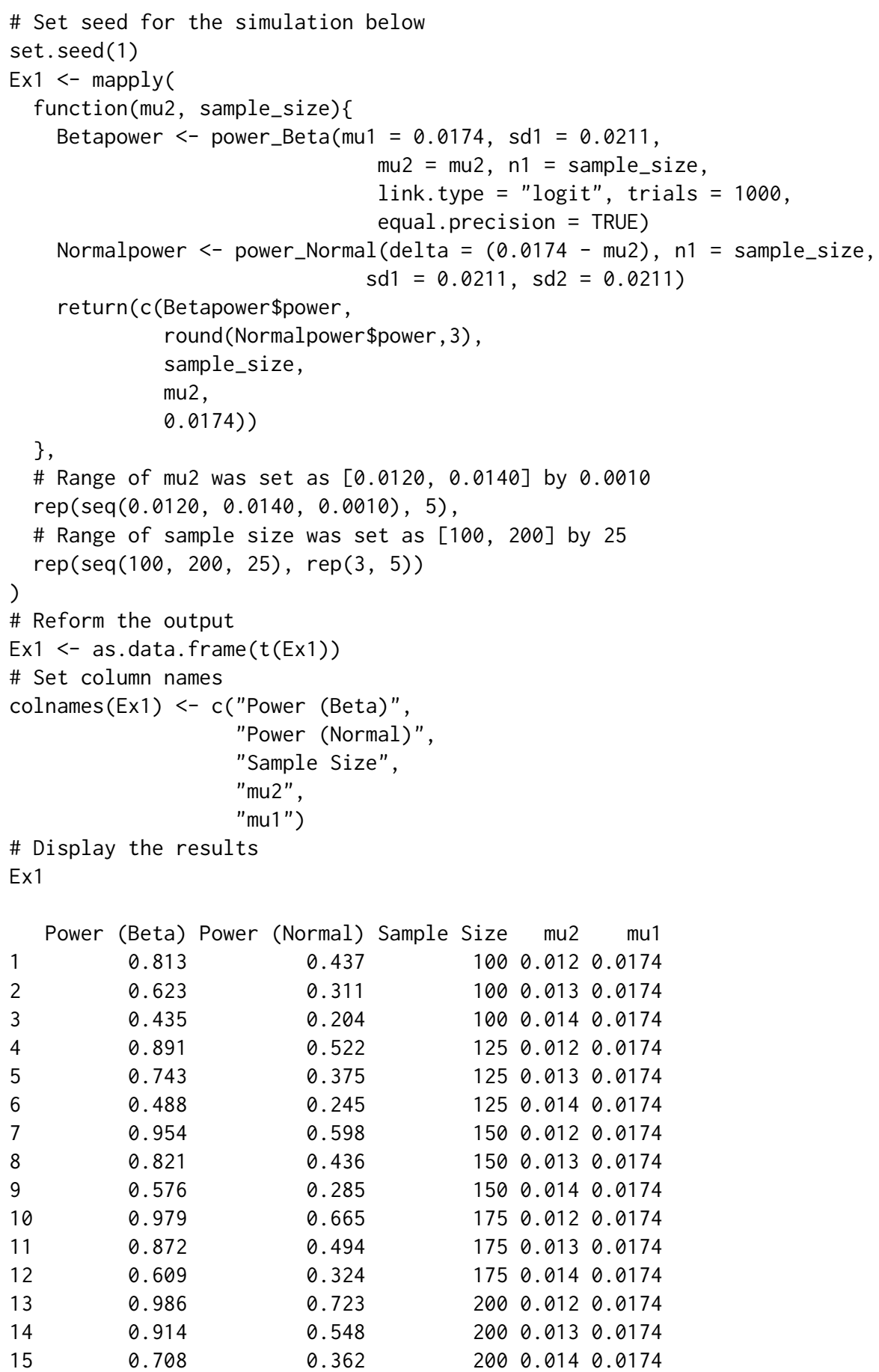

When equal precision cannot be assumed, equal. precision is set to FALSE, and an input value for $\mathrm{sd} 2$ is required. To demonstrate unequal precision, the previous example is rerun with equal . precision=FALSE and $s d 2=0.03$. The output is provided below.

\# Set seed for the simulation below 


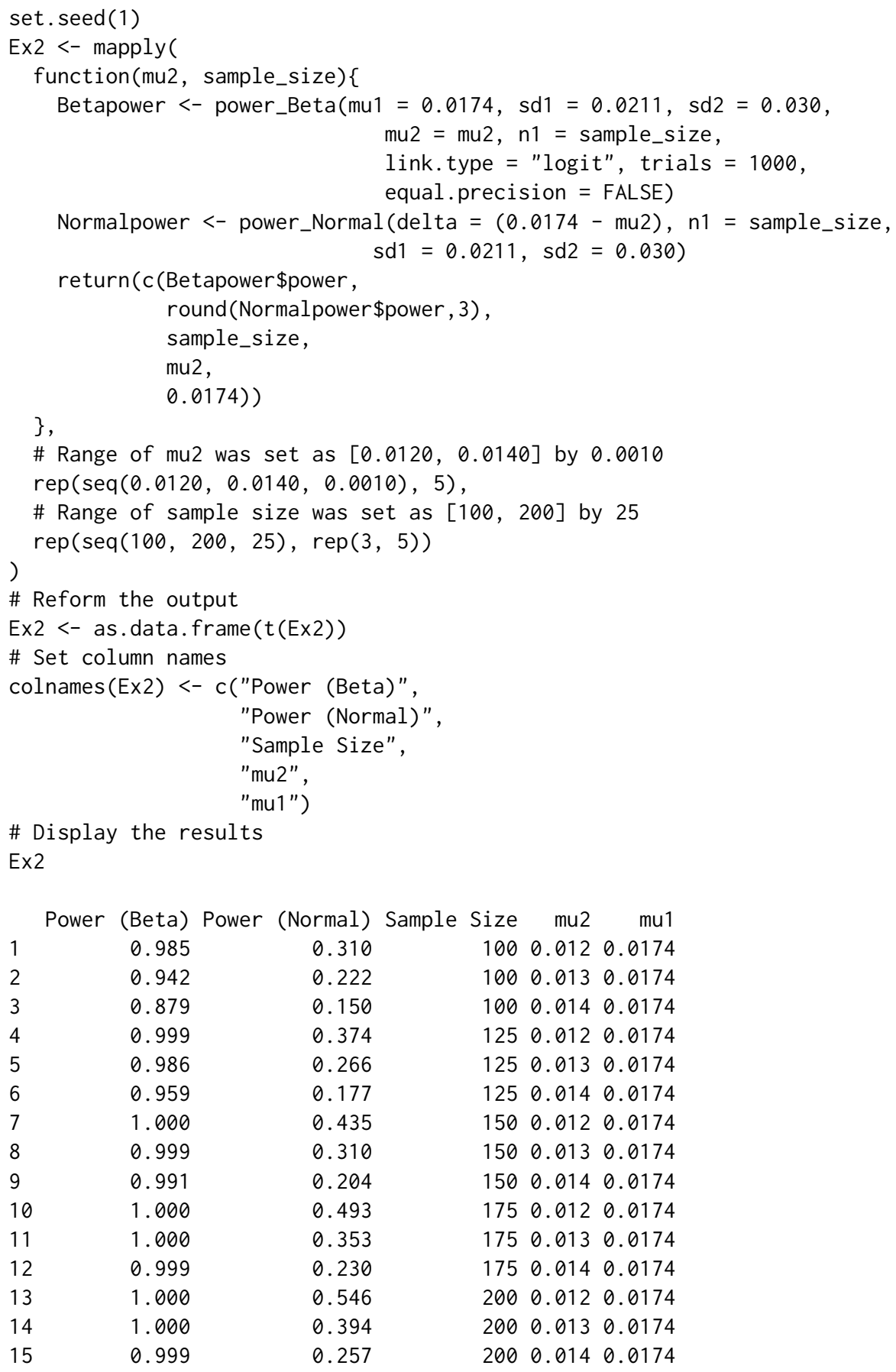

The results indicate small differences between the power of a two-sided $t$-test with equal and unequal standard deviations, while the power from power_Beta changes drastically without the equal precision assumption. Unlike normally distributed random variables, the beta distribution is more sensitive to the assumption of equal precision parameters. Figure 1 displays the comparison of probability density functions for beta distributed random variables with and without the equal precision assumption and the comparison for the analogous normally distributed variables with and without equal standard deviations.

\section{Summary}

This example demonstrates the use of power_Beta and power_Normal, each with equal and unequal precision parameters, to perform power analyses and sample size calculations. Since a simulation method is used within the function power_Beta, the computational time is dependent upon the number of trials in the simulation. It is suggested that a starting value be used, such as 100 , to determine an initial range for the other parameters (e.g., range of mu2). Once an appropriate range of values 
equal precision or standard deviation

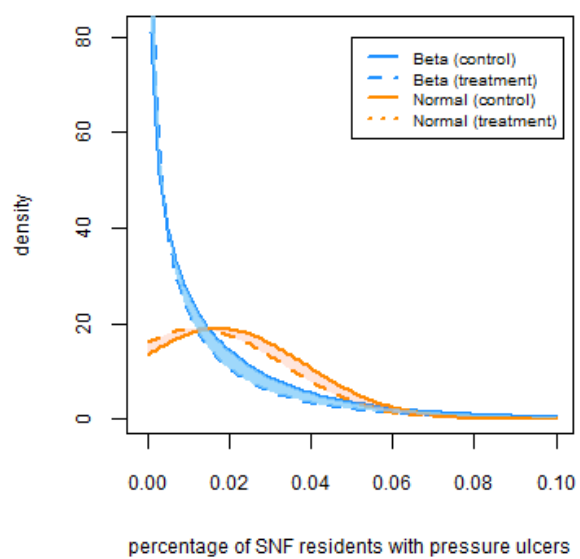

unequal precision or standard deviation

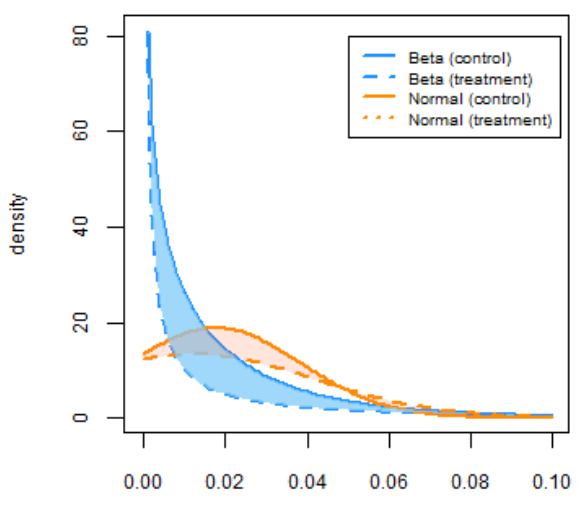

percentage of SNF residents with pressure ulcers

Figure 1: Comparison of equal and unequal precision or standard deviation parameters (mu2 is assumed to be 0.0120).

is determined, the number of trials should be increased (e.g., trials=1000) to output more precise power and sample size estimates.

\section{Summary and Discussion}

Multiple packages are available in R to perform power analyses, including pwr, MESS, WebPower, and the base $\mathrm{R}$ stats package. However, these packages do not provide a comprehensive power analysis toolkit capable of calculating power or sample sizes for the test of two-sample means or ratios when the outcomes have a beta, gamma, or Poisson distribution.

The PASSED package extends the current power analysis functions available in R. Seven functions are provided for corresponding distributions, applying either theoretical formulas or simulation algorithms. All functions have the ability to obtain the statistical power or estimate minimum sample sizes. In particular, the formula-based approaches also support calculations for other parameters such as means and proportions. As for the simulation-based methods, users are able to customize each analysis with options to set the number of trials in the simulation and specify the assumptions for the tests. An example of how to implement and customize the functions is provided in Section Application of PASSED. The PASSED package provides a simple, one-package solution for sample size and power calculations for a wide variety of common and specialty distributions encountered in clinical research.

\section{Computational Details}

The results in this paper were obtained using $\mathrm{R}$ 4.0.2 and betareg 3.0.0. $\mathrm{R}$ itself and all packages used are available from the Comprehensive R Archive Network (CRAN) at https: //CRAN. R-project. org/.

\section{Acknowledgments}

The authors gratefully acknowledge Dr. Richard Madsen, Professor Emeritus of Statistics University of Missouri - Columbia, for his contribution and interest in this topic. Funding for this project was provided by NIH R34AR074209A (Co-I Leary) and the Thompson Laboratory for Regenerative Orthopaedics, University of Missouri - Columbia. 


\section{Appendix}

\section{Derivation of Power Calculation Formulae for Poisson Distribution}

Let $x_{i j}$ be the number of events during the necessary study time $t_{i}$ from the $j$ th subject in the ith treatment group, $j=1, \ldots, n_{i}, i=1,2$. It is assumed that $x_{i j}$ are Poisson random variables with rate $\lambda_{i}$ such that the probability function of $x_{i j}$ is

$$
P\left(x_{i j}\right)=\frac{t_{i} \lambda_{i} e^{t_{i} \lambda_{i}}}{x_{i j} !}
$$

where $i=1,2$. Then, the total number of events in each group, denoted as $X_{1}$ and $X_{2}$, also follows a Poisson distribution:

$$
X_{i} \sim \operatorname{Poisson}\left(\lambda_{i} t_{i} n_{i}\right)
$$

For the hypothesis tests:

$$
H_{0}: \lambda_{2} / \lambda_{1}=R \text { vs. } H_{a}: \lambda_{2} / \lambda_{1}=R^{\prime}>R(\text { one }- \text { sided })
$$

and

$$
H_{0}: \lambda_{2} / \lambda_{1}=R \text { vs. } H_{a}: \lambda_{2} / \lambda_{1}=R^{\prime} \neq R(\text { two }- \text { sided }),
$$

where $R$ denotes the pre-specified ratio of two Poisson rates. Gu et al. (2008) derives a test statistic $W_{5}$, which is asymptotically distributed as a standard normal under the null hypothesis above,

$$
W_{5}=\frac{2\left(\sqrt{X_{2}+3 / 8}-\sqrt{Q\left(X_{1}+3 / 8\right)}\right)}{\sqrt{1+Q}},
$$

where $Q=R / d$ and $d=t_{1} / t_{2}$. Then, the critical region of the one-sided test is

$$
W_{5}=\frac{2\left(\sqrt{X_{2}+3 / 8}-\sqrt{Q\left(X_{1}+3 / 8\right)}\right)}{\sqrt{1+Q}} \geq z_{1-\alpha} .
$$

To calculate the power under $H_{a}: \lambda_{2} / \lambda_{1}=R^{\prime}>R$ at significance level $\alpha$, let $c=R / R^{\prime}$ and multiply both sides of Equation 4 by $\sqrt{1+Q}$, which is greater than 0 as that

$$
2\left(\sqrt{X_{2}+3 / 8}-\sqrt{Q\left(X_{1}+3 / 8\right)}\right) \geq z_{1-\alpha} \sqrt{1+Q} .
$$

Add $-2(\sqrt{Q / c}-\sqrt{Q}) \sqrt{X_{1}+3 / 8}$ to both sides of Equation 5 for the inequality,

$$
2\left(\sqrt{X_{2}+3 / 8}-\sqrt{Q / c\left(X_{1}+3 / 8\right)}\right) \geq z_{1-\alpha} \sqrt{1+Q}-2(\sqrt{Q / c}-\sqrt{Q}) \sqrt{X_{1}+3 / 8} .
$$

Then, divide both sides of Equation 6 by $\sqrt{1+Q / c}$, greater than 0 . It follows that

$$
\frac{2\left(\sqrt{X_{2}+3 / 8}-\sqrt{Q / c\left(X_{1}+3 / 8\right)}\right)}{\sqrt{1+Q / c}} \geq \frac{z_{1-\alpha} \sqrt{1+Q}-2(\sqrt{Q / c}-\sqrt{Q}) \sqrt{X_{1}+3 / 8}}{\sqrt{1+Q / c}} .
$$

Under the alternative hypothesis, the left-hand side of Equation 7 is asymptotically normal distributed (Gu et al., 2008). Accordingly, the type II error, $\beta$, can be derived as:

$$
\begin{aligned}
\beta & =P\left(H_{0} \mid H_{a}\right) \\
& =P\left(X<\frac{z_{1-\alpha} \sqrt{1+Q}-2(\sqrt{Q / c}-\sqrt{Q}) \sqrt{X_{1}+3 / 8}}{\sqrt{1+Q / c}} \mid H_{a}\right) \\
& =\Phi\left(\frac{z_{1-\alpha} \sqrt{1+Q}-2(\sqrt{Q / c}-\sqrt{Q}) \sqrt{X_{1}+3 / 8}}{\sqrt{1+Q / c}}\right)
\end{aligned}
$$


Incorporating $Q=R / d, c=R / R^{\prime}$ and $X_{1}=\lambda_{1} t_{1} n_{1}$, and collecting items

$$
\begin{aligned}
\beta & =\Phi\left(\frac{z_{1-\alpha} \sqrt{1+R / d}-2\left(\sqrt{(R / d) /\left(R / R^{\prime}\right)}-\sqrt{R / d}\right) \sqrt{\lambda_{1} t_{1} n_{1}+3 / 8}}{\sqrt{1+(R / d) /\left(R / R^{\prime}\right)}}\right) \\
& =\Phi\left(\frac{z_{1-\alpha} \sqrt{\frac{R+d}{d}}-2\left(\sqrt{\frac{R^{\prime}}{d}}-\sqrt{\frac{R}{d}}\right) \sqrt{\lambda_{1} t_{1} n_{1}+3 / 8}}{\sqrt{\frac{R^{\prime}+d}{d}}}\right) \\
& =\Phi\left(\frac{z_{1-\alpha} \sqrt{\frac{R+d}{R^{\prime}}}-2\left(1-\sqrt{\frac{R}{R^{\prime}}}\right) \sqrt{\lambda_{1} t_{1} n_{1}+3 / 8}}{\sqrt{\frac{R^{\prime}+d}{R^{\prime}}}}\right) \\
& =\Phi\left(\frac{z_{1-\alpha} C-A \sqrt{B}}{D}\right),
\end{aligned}
$$

where $A=2\left(1-\sqrt{\frac{R}{R^{\prime}}}\right)$,

$B=\lambda_{1} t_{1} n_{1}+3 / 8$,

$C=\sqrt{\frac{R+d}{R^{\prime}}}$

$D=\sqrt{\frac{R^{\prime}+d}{R^{\prime}}}$

So, power can be expressed as

$$
\begin{aligned}
\operatorname{Power}\left(W_{5}\right) & =1-\Phi\left(\frac{z_{1-\alpha} C-A \sqrt{B}}{D}\right) \\
& =\Phi\left(\frac{A \sqrt{B}-z_{1-\alpha} C}{D}\right) .
\end{aligned}
$$

Moreover, using $z_{\text {power }}=\Phi^{-1}($ Power $)$, Equation 8 can be expressed as:

$$
z_{\text {power }}=\frac{A \sqrt{B}-z_{1-\alpha} C}{D} \text {. }
$$

Solving Equation 9 for $B$,

$$
B=\left(\frac{z_{\text {power }} D-z_{1-\alpha} C}{A}\right)^{2}
$$

Since $B=\lambda_{1} t_{1} n_{1}+3 / 8$, the sample size calculation formula of one-sided test can be determined by solving Equation 10 for $n_{1}$

$$
n_{1}=\frac{\left(\frac{z_{1-\alpha} C+z_{\text {power }} D}{A}\right)^{2}-\frac{3}{8}}{\lambda_{1} t_{1}}(\text { one }- \text { sided })
$$

and the two-sided test can be derived similarly as

$$
n_{1}=\frac{\left(\frac{z_{1-\frac{\alpha}{2}} C+z_{\text {power }} D}{A}\right)^{2}-\frac{3}{8}}{\lambda_{1} t_{1}}(\text { two }- \text { sided }) .
$$

\section{Bibliography}

C. L. Aberson. Applied power analysis for the behavioral sciences. Routledge, 2019. URL https://doi . org/10.4324/9781315171500. [p542]

E. Brittain and J. J. Schlesselman. Optimal allocation for the comparison of proportions. Biometrics, pages 1003-1009, 1982. URL https://doi.org/10.2307/2529880. [p543]

G. Casella and R. L. Berger. Statistical inference, volume 2. Duxbury Pacific Grove, CA, 2002. ISBN 978-0-357-75313-2. [p550]

S. Champely, C. Ekstrom, P. Dalgaard, J. Gill, S. Weibelzahl, A. Anandkumar, C. Ford, R. Volcic, and H. De Rosario. pwr: Basic functions for power analysis, 2017. URL https: //cran. r-project. org/ web/packages/pwr/index.html. [p542, 548]

C.-H. Chang, J.-J. Lin, and N. Pal. Testing the equality of several gamma means: a parametric bootstrap method with applications. Computational Statistics, 26(1):55-76, 2011. URL https://doi . org/10. 1007/s00180-010-0209-1. [p551] 
M. R. Chernick and C. Y. Liu. The saw-toothed behavior of power versus sample size and software solutions: Single binomial proportion using exact methods. The American Statistician, 56(2):149-155, 2002. URL https://doi.org/10.1198/000313002317572835. [p550]

S.-C. Chow, H. Wang, and J. Shao. Sample size calculations in clinical research. Chapman and Hall/CRC, 2007. URL https://doi.org/10.1002/wics.155. [p542]

N. Cressie and H. Whitford. How to use the two sample t-test. Biometrical Journal, 28(2):131-148, 1986. URL https://doi .org/10.1002/bimj . 4710280202. [p547]

F. Cribari-Neto and A. Zeileis. Beta regression in R. Journal of Statistical Software, 34(2):1-24, 2010. URL https://doi.org/10.18637/jss.v034.i02. [p549]

M. D. Huffman. An improved approximate two-sample poisson test. Journal of the Royal Statistical Society: Series C (Applied Statistics), 33(2):224-226, 1984. URL https://doi .org/10.2307/2347448. [p546]

C. T. Ekstrøm. The R primer. CRC Press USA, 2012. URL https://doi .org/10.1201/9781315154411. [p542, 548]

S. Ferrari and F. Cribari-Neto. Beta regression for modelling rates and proportions. Journal of Applied Statistics, 31(7):799-815, 2004. URL https://doi .org/10.1080/0266476042000214501. [p549]

J. L. Fleiss, A. Tytun, and H. K. Ury. A simple approximation for calculating sample sizes for comparing independent proportions. Biometrics, pages 343-346, 1980. URL https: //doi . org/10. 2307/2529990. [p543]

S. Gates, I. Nguyen, M. Del Core, P. A. Nakonezny, H. Bradley, and M. Khazzam. Incidence and predictors of positive intraoperative cultures in primary shoulder arthroplasty following prior ipsilateral shoulder surgery. JSES International, 2020. URL https://doi.org/10.1016/j.jseint. 2019.12.011. [p544]

K. Gu, H. K. T. Ng, M. L. Tang, and W. R. Schucany. Testing the ratio of two poisson rates. Biometrical Journal: Journal of Mathematical Methods in Biosciences, 50(2):283-298, 2008. URL https: //doi .org/ 10.1002/bimj.200710403. [p546, 547, 556]

A. K. Gupta and S. Nadarajah. Handbook of Beta Distribution and Its Applications. CRC Press, New York, 2004. URL https://doi.org/10.1201/9781482276596. [p548]

J. M. Hilbe. Negative binomial regression. Cambridge University Press, 2011. ISBN 978-0-521-19815-8. [p544]

Y.-R. Hong, R. G. Salloum, S. Yadav, G. Smith, and A. G. Mainous III. Patient-provider discussion about cancer treatment costs and out-of-pocket spending: Implications for shared decision making in cancer care. Value in Health, 2020. URL https://doi .org/10.1016/j. jval .2020.08.002. [p550]

C. Hu, H. Zhou, and A. Sharma. Application of beta-distribution and combined uniform and binomial methods in longitudinal modeling of bounded outcome score data. The AAPS Journal, 22, 2020. URL https://doi.org/10.1208/s12248-020-00478-5. [p548]

M. K. Hutchinson and M. C. Holtman. Analysis of count data using poisson regression. Research in nursing $\mathcal{E}$ health, 28(5):408-418, 2005. URL https://doi .org/10.1002/nur. 20093. [p546]

S.-L. Jan and G. Shieh. Optimal sample sizes for welch's test under various allocation and cost considerations. Behavior research methods, 43(4):1014-1022, 2011. URL https: // doi . org/10.3758/ s13428-011-0095-7. [p548]

S. Jones, S. Carley, and M. Harrison. An introduction to power and sample size estimation. Emergency Medicine Journal, 20(5):453-458, 2003. URL https://doi .org/10.1136/emj .20.5.453. [p542]

M. Kohl. MKmisc: Miscellaneous functions from M. Kohl, 2021. URL https: / / www. stamats. de. R package version 1.8. [p542]

B. LeBeau. Power Analysis by Simulation using R and simglm, 2019. URL https://doi .org/10.17077/ f7kk-6w7f. [p542]

C. B. G. LEITE, L. V. Ranzoni, P. N. Giglio, M. B. Bonadio, L. D. P. Melo, M. K. Demange, and R. G. Gobbi. Assessment of the use of tranexamic acid after total knee arthroplasty. Acta Ortopédica Brasileira, 28(2):74-77, 2020. URL https://doi .org/10.1590/1413-785220202802228410. [p543] 
C. Luan, D.-T. Xu, N.-J. Chen, F.-F. Wang, K.-S. Tian, C. Wei, and X.-B. Wang. How to choose kinematic or mechanical alignment individually according to preoperative characteristics of patients? BMC Musculoskeletal Disorders, 21(1):1-6, 2020. URL https://doi .org/10.1186/s12891-020-03472-2. [p547]

K. Pearson. X. on the criterion that a given system of deviations from the probable in the case of a correlated system of variables is such that it can be reasonably supposed to have arisen from random sampling. The London, Edinburgh, and Dublin Philosophical Magazine and Journal of Science, 50 (302):157-175, 1900. URL https://doi .org/10.1080/14786440009463897. [p543]

D. Plessl, B. Salomon, A. Haydel, C. Leonardi, A. Bronstone, and V. Dasa. Rapid versus standard recovery protocol is associated with improved recovery of range of motion 12 weeks after total knee arthroplasty. The Journal of the American Academy of Orthopaedic Surgeons, 2020. URL https: //doi.org/10.5435/JAAOS-D-19-00597. [p542, 546]

R Core Team. R: A Language and Environment for Statistical Computing. R Foundation for Statistical Computing, Vienna, Austria, 2016. URL https: / /www. R-project. org/. ISBN 3-900051-07-0. [p542, 550]

Scherer. Package 'samplesize', 2016. URL https://cran.r-project.org/web/packages/samplesize/ index.html. [p542]

P. T. Schickedanz and G. F. Krause. A test for the scale parameters of two gamma distributions using the generalized likelihood ratio. Journal of applied meteorology, 9(1):13-16, 1970. URL https: //doi.org/10.1175/1520-0450(1970)009<0013:ATFTSP>2.0.c0;2. [p552]

W.-K. Shiue and L. J. Bain. Experiment size and power comparisons for two-sample poisson tests. Journal of the Royal Statistical Society: Series C (Applied Statistics), 31(2):130-134, 1982. URL https: //doi.org/10.2307/2347975. [p546]

W.-K. Shiue and L. J. Bain. A two-sample test of equal gamma distribution scale parameters with unknown common shape parameter. Technometrics, 25(4):377-381, 1983. URL https://doi .org/10. 1080/00401706.1983.10487901. [p551]

W.-K. Shiue, L. J. Bain, and M. Engelhardt. Test of equal gamma-distribution means with unknown and unequal shape parameters. Technometrics, 30(2):169-174, 1988. URL https: //doi . org/10.1080/ 00401706.1988.10488364. [p551]

M. Smithson and J. Verkuilen. A better lemon squeezer? maximum-likelihood regression with betadistributed dependent variables. Psychological Methods, 11(1):54-71, 2006. URL https: //doi .org/ 10.1037/1082-989X.11.1.54. [p549]

H. C. Thode. Power and sample size requirements for tests of differences between two poisson rates. Journal of the Royal Statistical Society: Series D (The Statistician), 46(2):227-230, 1997. URL https://doi.org/10.1111/1467-9884.00078. [p546]

E. Zhang, V. Q. Wu, S.-C. Chow, and H. G. Zhang. Package 'TrialSize', 2013. URL https://cran.rproject.org/web/packages/TrialSize/index.html. [p542]

Z. Zhang and Y. Mai. WebPower: Basic and Advanced Statistical Power Analysis, 2018. URL https: //CRAN.R-project.org/package=WebPower. R package version 0.5.2. [p542]

H. Zhu and H. Lakkis. Sample size calculation for comparing two negative binomial rates. Statistics in medicine, 33(3):376-387, 2014. URL https://doi .org/10.1002/sim.5947. [p544, 545]

Jinpu $\mathrm{Li}$

University of Missouri

USA

E-mail: lijinpahealth.missouri.edu

Ryan P. Knigge

University of Minnesota

USA

E-mail: knigg008@umn.edu 
Kaiyi Chen

University of Missouri

USA

E-mail: kcvpc@mail.missouri.edu

Emily V. Leary, Ph.D.

Thompson Laboratory for Regenerative Orthopaedics

Department of Orthopaedic Surgery

University of Missouri

USA

E-mail: learye@health.missouri.edu 